\title{
Guest Editorial: Convergence and Advanced Technology
}

\author{
Sang-Yeob Oh • Supratip Ghose • Joong-Kyung Ryu
}

Published online: 3 March 2015

(C) Springer Science+Business Media New York 2015

Convergence and advanced technologies spring forth as a result of lateral entry by firms in different markets, and today, there is a synergistic combination of traditional industries and state-of-the-art technologies, such as information technology. The main goal of this issue is to bring researchers from academia together with professionals from different industries to share algorithms, solutions, and architectures as well as their experiences. We expect this special issue to be viewed as a continuum of products and as the lateral entry of research from different technological perspectives on digital management. The effort diffuses technological competencies and values within existing technologies' base information systems. The convergence process blurs traditional technological and sectorial boundaries and thus opens new avenues of technological innovation in ubiquitous technologies $[1,4,6,10,15]$. This issue challenges processing overhead in convergence and advanced technologies.

The paper by Kim et al. [5] introduces an efficient mobility management scheme for convergence of mobile media multicast services in next-generation networks. The proposed scheme applies a method to quickly rejoin a relevant multicast stream by enabling the AIMSMM system to collect multicast group information through a multicast group information handler located in the multicast router. The paper by Oh et al. [2] presents the development of a one-source multi-use cross-platform based on zero coding. This method is to research and develop a native application-based development tool that can perfectly satisfy both onesource-multi-use and cross-platform, and secure stable hardware control and execution speed.

The paper by Je-Ho Park [19] presents low-cost image indexing for a massive database. The proposed method is an identifier generation for indexing that is efficient and effective from the perspective of cost and indexing performance, in order to compose identifiers with high cardinality. The paper by Jo et al. [9] presents an access control system for secure extensible markup language (XML) telemedicine documents. This

S.-Y. Oh

Department of Interactive Media, Gachon University, Seongnam, Republic of Korea

e-mail: syoh1234@gmail.com

S. Ghose

Department of Computer Science and Engineering, University of Information Technology \& Sciences,

Dhaka, Bangladesh

e-mail: sgresearch@gmail.com

J.-K. Ryu ( $\bowtie)$

Department of Computer Software, Daelim University, Anyang, Republic of Korea

e-mail: jkryu.hci@gmail.com 
paper designs and builds an XML Access Control System (XACS) that is capable of making fined-grained access control. This only provides data corresponding to its users' authority levels by authorizing them to access only specific items when they are searching XML documents in a telemedicine health service.

The paper by Park et al. [7] proposes Linked Tag, a semi-automatic image annotation system that inserts semantic relationships between tags. The proposed annotation method connects image tags using predicate words that can capture the context in which the image tags are used. The main idea is that by bridging semantic links, the difficulty in determining the meaning of the tags can be overcome. The paper by Lee et al. [18] proposes a novel imagesearching scheme that extracts image features using a combination of Advanced Speed-Up Robust Feature (ASURF) and Dominant Color Descriptor (DCD). The system (for mobile image search) runs on the iPhone and can easily be used to find and describe a natural color image. The authors' main contribution is high accuracy and stability from using ASURF and DCD to search for natural images on mobile platforms.

The paper by Chung et al. [3] presents a dynamic binary analyzer that can find unknown vulnerabilities and self-modifying code. They adopt taint analysis to find vulnerabilities that transpire during runtime. Adopting dynamic analysis that drives and analyzes the system only under virtual machine circumstances through an emulator can help detect falsification of program code. The paper by Lim et al. [25] proposes feature selection for daily peak load forecasting using a neuro-fuzzy system. This paper presents a Neuro-fuzzy-based Daily Peak Load Forecasting (N-DPLF) model with a feature-selection method for DPLF. Nine common features were extracted using average slope, position, and direct methods. The load data were clustered into seven subsets according to season and day type. The features with the highest salience ranks out of the nine extracted features were selected by NADM.

The paper by Lee et al. [21] presents design patterns for context-aware services. This paper presents a novel programming model to enable the end user to build context-aware services. The concept of programming by selection and its prototype implementation are presented. Selection offers an alternative end-user programming method for context-aware services. The paper by Kwon et al. [22] introduces error correction for a reference indexing system including multimedia journals. The types of reference listings are analyzed, and the major types are categorized. Then, the correction lookup table and indices are built to calculate more accurate impact factors. The actual impact factors and immediacy indices for the journals listed in the database are calculated.

The paper by Shin et al. [11] proposes an intuitive user interaction method using multisensors in non-photorealistic rendering. The proposed method renders a pencil drawing filter for a given photo image with the direction and strength of line strokes that are applied in vector fields. The method changes the direction of the stroke with $\mathrm{x}$-coordinate values and $\mathrm{y}$ coordinate values and controls the strength of the stroke with $\mathrm{z}$-coordinate values after catching three coordinate values in real-time from the orientation sensor on a mobile device. The paper by Choi et al. [23] shows a comparison of knowledge, attitudes, and trust for the use of personal health information in clinical research. They determined the knowledge, attitudes and levels of trust towards the use of personal health information in clinical research, focusing on three groups. The three groups had significantly different attitudes about, and trust towards, the use of protected health information.

The paper by Joon Yoo [26] proposes a new receiver-centric media access control protocol, called RIMAC, to increase spatial reuse and network capacity. In RIMAC, the receivers accurately assess their channel status, as opposed to the sender-initiated approach where senders can only heuristically infer the status of their receivers. The receiver-centric approach achieves a better level of spatial reuse with a degree of 
interference prevention. The paper by Chulyun Kim [14] introduces and analyzes the MAVELET algorithm, which constructs a whole wavelet synopsis on distributed data sets. Wavelet synopsis is one of the most popular dimensionality reduction methods, and MapReduce is the most popular distributed platform, with good scalability and fault tolerance. Thus, it is very valuable to study the algorithm to construct the wavelet synopsis in the MapReduce framework. The proposed algorithm consists of a single MapReduce phase and the amount of data communicated through the network of the distributed MapReduce platform is significantly reduced.

The paper by Jeong [8] presents social networking site-based recommendation mechanisms for social media. This is a recommendation algorithm based on the intimacy between users, which is proposed using information on the social network that correlates with the user. The proposed method was applied to podcasts in the form of a multimedia file to be implemented as a system, demonstrated through experiment results. The paper by Chung et al. [13] introduces an interactive pain nursing intervention system for smart health services, which supports effective and linked performance in sign assessment, pain diagnosis, nursing intervention, and implementation for input of information on new patients.

The paper by Park et al. [12] develops a u-healthcare monitoring system that offers an alarm service for measurement, and notification in case of unusual levels of patient indicators, based on the user's bio-signal data and context-awareness. This provides a more accurate measurement value than previous monitoring services because it considers each user's condition, and can reduce the user's health risks using the patient's life management and lifestyle measurement values. The paper by Choi et al. [24] presents users' perceptions on telemedicine services: a comparative study of public/private healthcare. They conducted a comparative study focusing on public healthcare and private healthcare to determine how users perceive telemedicine services differently. They performed two independent sample t-tests, depending on the group, to measure differences in satisfaction and continuous intention to use the services, as well as perception of the telemedicine service.

The paper by Kim et al. [17] proposes a large-scale object recognition system using publicly available software. The proposed system's performance with the current MPEG CDVS standard was evaluated by using a database containing two-dimensional planar object datasets of categories with one million distracter images. The authors found characteristics of the vocabulary tree, limiting adaptation to a specific application domain. The paper by Park et al. [20] proposes a picocell-based telemedicine health service for human UX/UI based on BT-IT fusion technology considering user convenience. The medical service is a telemedicine health service that can overcome the spatial limitations of hospital-oriented medical services in order to improve user convenience while naturally combining life and medical service spaces. The paper by Lee et al. [16] introduces the effects of transportation energy policy on fuel consumption and transportation safety. This paper examined the impact of transportation energy policies on traffic safety through policy simulations. Considering the changes in vehicle miles traveled and in vehicle stock composition as a result of policy changes, the impact of these changes on traffic accidents was examined in terms of the number of traffic accidents, the number of traffic fatalities, and total accident costs.

This fine collection of papers was achieved by fruitful collaboration. We wish to thank all the authors for their contributions and the reviewers for assisting our editorial work. We do hope that the papers included in this special issue will satisfy the audience of Multimedia Tools and Applications and readers will find them interesting. Furthermore, we would like to thank Prof. Borko Furht, Editor-in-Chief of Multimedia Tools and Applications, for his valuable remarks and his undeterred help throughout the publication process of this special issue. 


\section{References}

1. Boutaba R, Chung K, Gen M (2014) Recent trends in interactive multimedia computing for industry. Clust Comput. doi:10.1007/s10586-014-0349-0

2. Chang Y, Oh SY (2014) A study on the development of one source multi Use cross-platform based on zero coding. Multimedia Tools and Applications. doi:10.1007/s11042-014-1886-5

3. Choi YH, Park MW, Eom JH, Chung TM (2014) Dynamic binary analyzer for scanning vulnerabilities with taint analysis. Multimedia Tools and Applications. doi:10.1007/s11042-014-1922-5

4. Chung K (2014) Recent Trends on Convergence and Ubiquitous Computing. Pers Ubiquit Comput 18(6)

5. Gu I, Ryu W, Kim J (2014) An efficient mobility management scheme for convergence mobile media multicast services in NGN. Multimedia Tools and Applications. doi:10.1007/s11042-014-1996-0

6. Han J (2014) Distributed hybrid P2P networking systems. Peer-to-Peer Networking and Applications. doi: 10.1007/s12083-014-0298-7

7. Im DH, Park GD (2014) Image annotation using semantic relationships between image tags. Multimedia Tools and Applications. doi:10.1007/s11042-014-1855-Z

8. Jeong OR (2014) SNS-based recommendation mechanisms for social media. Multimedia Tools and Applications. doi:10.1007/s11042-014-1884-7

9. Jo SM, Chung KY (2014) Design of access control system for telemedicine secure XML documents. Multimedia Tools and Applications. doi:10.1007/s11042-014-1938-X

10. Jung H, Chung K (2013) Mining based associative image filtering using harmonic mean. Clust Comput. doi: 10.1007/s10586-013-0318-Z

11. Jung JJ, Kim JY, Chung HS, Shin PS (2014) An intuitive user interaction method using multi-sensors for pencil drawing filter of NPR rendering in mobile devices. Multimedia Tools and Applications. doi:10.1007/ s11042-014-2054-7

12. Jung EY, Kim JT, Soh J, Park DK (2014) Development of U-healthcare monitoring system based on contextaware for knowledge service. Multimedia Tools and Applications. doi:10.1007/s11042-014-2037-8

13. Jung H, Yoo H, Lee YH, Chung KY (2014) Interactive pain nursing intervention system for smart health service. Multimedia Tools and Applications. doi:10.1007/s11042-014-1923-4

14. Kim C (2014) Theoretical analysis of constructing wavelet synopsis on partitioned data sets. Multimedia Tools and Applications. doi:10.1007/s11042-014-1908-3

15. Kim JH, Chung K (2014) Ontology-based healthcare context information model to implement ubiquitous environment. Multimedia Tools and Applications 71(2):873-888

16. Kim CK, Chung KY, Kim Y, Lee KD (2014) The effects of transportation energy policy on fuel consumption and transportation safety. Multimedia Tools and Applications. doi:10.1007/s11042-014-1974-6

17. Kim MU, Yoon K (2014) Performance evaluation of large-scale object recognition system using Bag-ofvisual words model. Multimedia Tools and Applications. doi:10.1007/s11042-014-2152-6

18. Lee YH, Kim Y (2014) Efficient image retrieval using advanced SURF and DCD on mobile platform. Multimedia Tools and Applications. doi:10.1007/s11042-014-2129-5

19. Park JH (2014) Low-cost image indexing for massive database. Multimedia Tools and Applications. doi:10. 1007/s11042-014-2026-y

20. Park RC, Jung H, Chung K, Yoon KH (2014) Picocell based telemedicine health service for human UX/UI. Multimedia Tools and Applications. doi:10.1007/s11042-014-1964-8

21. Park JK, Lee KH (2014) Design patterns for context-aware services. Multimedia Tools and Applications. doi:10.1007/s11042-014-2001-7

22. Park JH, Park HH, Kwon YB (2014) Error correction of reference indexing system including multimedia journals. Multimedia Tools and Applications. doi:10.1007/s11042-014-1971-9

23. Rho MJ, Jang KS, Chung KY, Choi IY (2014) Comparison of knowledge, attitudes, and trust for the Use of personal health information in clinical research. Multimedia Tools and Applications. doi:10.1007/s11042013-1772-6

24. Rho MJ, Yoon KH, Kim HS, Choi IY (2014) Users' perception on telemedicine service: a comparative study of public healthcare and private healthcare. Multimedia Tools and Applications. doi:10.1007/s11042-014-1966-6

25. Son SY, Lee SH, Chung K, Lim JS (2014) Feature selection for daily peak load forecasting using a neurofuzzy system. Multimedia Tools and Applications. doi:10.1007/s11042-014-1943-0

26. Yoo J (2014) Receiver-centric physical carrier sensing for vehicular Ad Hoc networks. Multimedia Tools and Applications. doi:10.1007/s11042-014-1851-3 


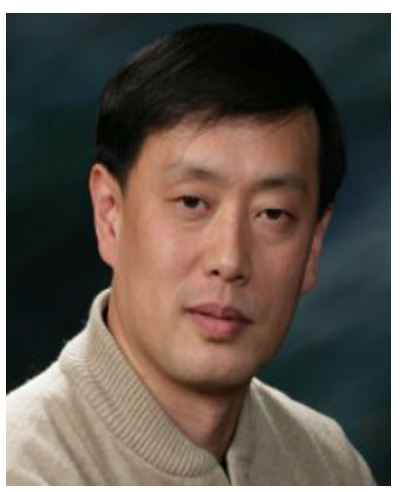

Sang-Yeob Oh received a BS in Computer Science from Kyungwon University in 1989 and an MS and a PhD from Kwangwoon University, Korea, in 1991 and 1999, respectively, from their departments of computer science. He is currently a professor in the Division of Computer Media, Gachon University, Korea. His research interests include Speech Recognition, Vehicle Safety Communications, and HCI. He has edited 27 computer science books. He serves as Executive Editing Director for the International Conference on Digital Policy Management, as Workshop Chair for the International Conference on Information Science and Application, and as Executive Editing Director on the Steering Committees for the International Conference on Convergence Technology. He is also an editorial member of the International Journal of Computer Virology and Hacking, among others.

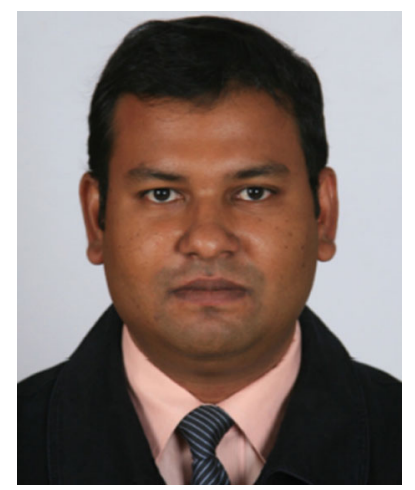

Supratip Ghose graduated from the Department of Applied Physics and Electronics, University of Rajshahi, with a BSc and an MSc in 1995 and 1998, respectively, and received his PhD in Information Engineering from the Department of Computer Science \& Information Engineering, Inha University, South Korea, in 2007. Dr. Ghose began his academic career as an Adjunct Lecturer at Inha University. After obtaining his $\mathrm{PhD}$, he served as an Assistant Professor in the Department of Computer Science and Engineering in UIC-BNU-HKBU in Zhuhai, China, at the University of Saint Joseph, Macau, China, and at Brac University in Dhaka, Bangladesh. He is currently an Associate Professor in the Department of Computer Science and Engineering, University of Information Technology \& Sciences, Dhaka, Bangladesh. His research interests include Data Mining, Collaborative Filtering, Sentiment Analysis in Social Webs, IR Frameworks, and Activity Recognition in Smartphones. 


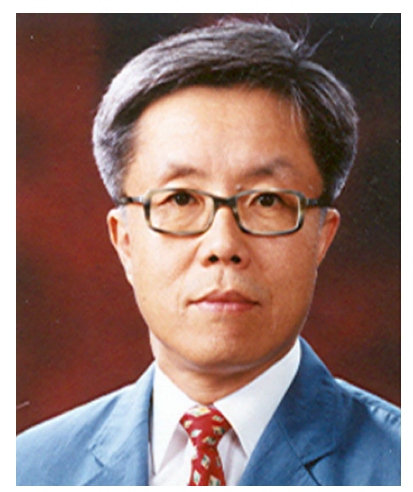

Joong-Kyung Ryu received a BS from the Department of Computer Science and Engineering, Korea National Open University, Korea, in 1988 and an MS from the Department of Information Engineering, Inha University, Korea in 1991. He received his $\mathrm{PhD}$ from the Department of Computer Information Engineering, Inha University, Korea in 2012. From 1983 to 1992, he was a researcher in the Department of Computer Center, Daelim Industrial Co., Ltd. He is currently a Professor in the Department of Computer Software, Daelim University, Korea. His research interests include Computer Architecture, u-Healthcare, Data Mining, and HCI. He serves as Executive Editing Director of the International Conference on IT Convergence and Security, on the Steering Committees of the International Conference on Convergence Technology. He is also an editorial board member for several international journals. 